\title{
The Research of Short-circuit Calculation and Optimization of Contain Distributed Power Distribution Network
}

\author{
Haiyang Yu ${ }^{1}$, Guangxin Zu ${ }^{1}$, Meilun Zhang ${ }^{1}$, Zhipeng Liu ${ }^{1}$, Guoliang Wu ${ }^{1}$, Jun Zheng ${ }^{1}$, and Wanlin Guan ${ }^{1}$ \\ ${ }^{1}$ Heilongjiang Electric Power Research Institute, Harbin 150030, China
}

\begin{abstract}
Electric power industry as an indispensable energy supply in daily life and production, which is the main motive force of equipment operation in the industrial times. Therefore, in order to meet the development needs and ensure the safety of power supply, it is necessary to optimize and transform the power grid. So that energy conservation and security of supply and demand can be ensured. In this paper, the short-circuit calculation and optimization design of distributed power distribution network are studied deeply, aiming at improving the performance of power grid and promoting the rapid development and transformation of power industry.
\end{abstract}

\section{Introduction}

Economic development depends on electricity, so doing a good job of grid security is the core topic which is faced by the current power industry. In this process, we should pay attention to the optimization of the original network, and constantly change the existing shortcomings to improve energy efficiency and reduce the occurrence of accidents as far as possible. There are many types of distributed powers which are widely used in the power grid. In order to reduce the safety accidents which are caused by short-circuit problems and ensure the high quality and efficiency of the power supply, the distribution network short-circuit calculation should be done very well to ensure the accurate, reasonable and effective calculation. And then based on the actual calculating results, the optimization can be more effective. In the course of research, according to the years of experience, the author summarized the relevant theores and the research results and gave the specific ideas for the corresponding problems, which lay the foundation for power grid optimization.

\section{The Introduction of the Distributed Power Supply}

In the power system, the distributed power supply is very common, we define it as the power supply which is relatively close to the user system and its capacity value is relatively small. The current social development should be based on sustainable development, with green, environmental protection, energy saving as the central idea. The power industry should also be so. Minimizing investment, reducing energy consumption and putting the distributed power into the large grid are the effective means to achieve this aim. In the course of the specific use, the setting of distributed power supply in the power distribution system will be changed because of the effect of the power supply type. For example, for the use of wind power in a power generation system, the synchronous generator or asynchronous generator will be used when the distributed power supply is connected to this system and the output form of the current is power frequency alternating current. For the power generation system which is composed of micro gas turbine, the permanent magnet generator is used mainly, and the output form of the current is high frequency alternating current. And in the course of output, the current is first converted into direct current by rectifier and then is converted into power frequency alternating current by inverter and finally enters into the power grid [1].

\section{The Type of Distributed Power Supply}

According to the actual investigation and the interface form of distributed power supply when it is connected to the distribution network, the distributed power supply can be divided into three kinds, one is based on the synchronous generator distributed power supply, the second one is based on the asynchronous generator distributed power supply, and the third one is based on the distributed power supply of PWM inverter. In the specific use process, according to the actual situation analysis, we should choose the appropriate access mode. Wind power technology has developed rapidly and has achieved a large number of grid-connected power generation. Among various renewable energy sources, wind power generation has the lowest cost and the least pollution, and it is one of the most widely distributed power generation technologies in the region. In 2009, in the "Emerging Energy Industry Development Plan" 
issued by the Chinese government, it was pointed out that the installed capacity of wind power should reach 130 million to 150 million kilowatts in the next decade. Wind power is a technology that converts wind energy into electricity. The wind turbine is mainly composed of wind turbines and wind turbines. Its output power is related to the wind power. Due to the randomness of wind energy, its output is unstable AC. In order to reduce the fluctuation of electric energy and ensure the quality of power supply, it must cooperate with the energy storage device. Use to suppress fluctuations in its output. Wind turbines generally convert mechanical energy into electrical energy through asynchronous generators to achieve grid-connected power generation. When voltage quality requirements are high, the inverter's unstable power can be integrated into $\mathrm{DC}$ by reverse rectifier power converter. The transformer converts the direct current into electrical energy that meets the user's requirements, thereby reducing the volatility of the wind energy and thereby improving the power quality.

Photovoltaic power generation uses the photovoltaic effect of semiconductor materials to convert solar energy directly into DC power without causing any chemical changes. The output power of photovoltaic power generation is related to the light intensity and the junction temperature of the battery. The power generation is uncontrollable. It sends electricity to the grid during the day and does not generate electricity at night. It has randomness and intermittentness, and its energy density is low. The actual power supply of a photovoltaic power generation system generally refers to a photovoltaic array. It is a DC power supply. Usually, the PWM inverter is used to convert the DC power into $\mathrm{AC}$ power and then connect to the power grid to supply power to the load. The volt-ampere characteristics of the PV array itself make it necessary to pass the maximum power regulation to achieve the desired operating efficiency. At the same time, in order to improve the safety and reliability of photovoltaic array grid-connected operation, the photovoltaic power generation system also needs the grid-connected control link, which controls the output characteristics of the power electronic inverter according to the actual operating parameters of the grid side. Photovoltaic arrays, maximum power controllers, power electronic converters, grid-connected controllers and other components constitute a complete photovoltaic grid-connected power generation system.

\section{Processing Methods and Optimization of Different Types of Distributed Power Supply}

The short-circuit calculation can be understood as the power flow calculation under the short-circuit condition. Based on the fault simulation circuit and the calculation of the power flow based on the Gaussian algorithm, the short-circuit current calculation of the distribution network with distributed power supply is realized. Firstly, the method needs to obtain the initial state of the distributed power supply through the steady-state power flow calculation based on the Gaussian algorithm, and then access the fault simulation circuit to modify the node impedance matrix used in the power flow calculation, according to whether the transient influence of the distributed power source is considered. The distributed power injection current value of different interface types is correctly calculated, so that the short-circuit power flow calculation is performed, thereby obtaining all the power flow information of the faulty network. In addition, in the short circuit calculation herein, the actions of various protection devices will not be considered to obtain the short circuit current value in the most severe case.

When the distributed power supply is connected to the power distribution system, in order to ensure the actual operational effect, the corresponding power equipment should be effectively evaluated and adjusted, so that the relay protection device action value can meet the actual needs. If the protective device in the current system can't satisfy the safety index, it is necessary to increase the corresponding security equipment. In this process, a specific adjustment should be made according to the short circuit current value, so we should pay more attention to the current calculation to ensure the accuracy of the calculation. In general, the main computational model is the steady state model of power flow computation. However, according to the actual investigation, when a short-circuit fault occurs, using this model will have a gap between the calculation result and actual situation. As for the simulation processing which takes the method of the connection of the ideal voltage source and the impedance, the calculation result and the real situation are still inconsistent. It belongs to a method of approximate treatment, so it still needs to be improved [2].

According to the above situation, a new calculating method will be put forward based on detailed learning about dynamic characteristics of all kinds of distributing power supply. When this method is used in the actual situation, firstly the output power of inverters, synchronous generator subtransient electromotive force and asynchronous generator subtransient electromotive force can be determined by power flow calculation. These can be used as reference values when the system short circuit is calculated. Next, processing method can be selected by displayed characteristics of all kinds of distributed power supply when they malfunction. The last step is short circuit fault simulation. The node voltage and fault current can be calculated by the combined mode of phase component and sequence component [3]

When photovoltaic power generation systems see fig 1 , battery and fuel cells are connected into grid systems, the inverters have to be used in the actual situation. During this process, the specific operational environment will influence power of inverters and energy characteristics of the power generation side. The hypothesis that the input power is constant can be drawn with fault analysis. The specific calculating method: the first step is that the power of inverters can be calculated by steady power flow calculation before the fault see fig 2. And then inverters need to be operated by a constant power after the fault. Related diagrams and computation formula are as follows: 


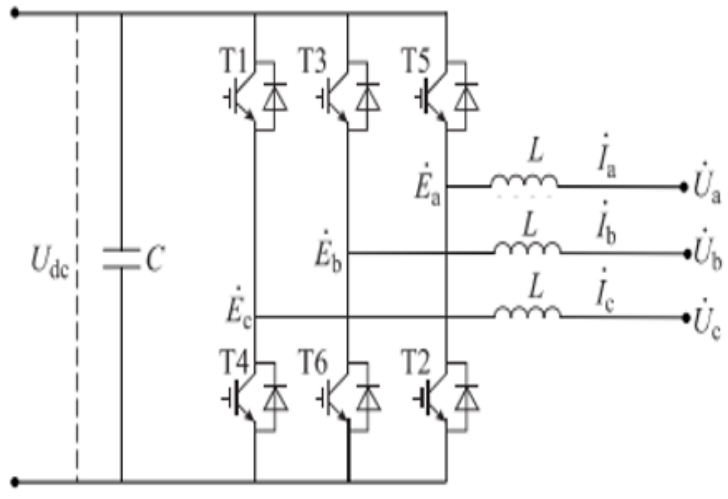

Figure 1. Inverter main circuit structure

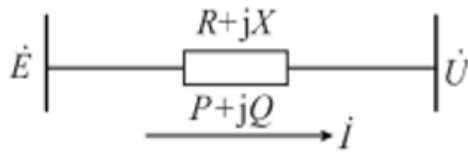

Figure 2. Inverter main circuit equivalent figure

The calculation of inverters internal positive sequence electromotive force $\dot{\mathrm{E}}$ based on existing relationship between voltage drop and power loss when short circuit fault happens.

$$
\begin{gathered}
\frac{P R+Q X}{U}=\Delta U \\
\frac{P X-Q R}{U}=\delta U \\
\dot{E}=U+\Delta U+j \delta U
\end{gathered}
$$

In this formula, $X$ refers to the reactance value of inverter output filter, $\mathrm{R}$ refers to the value of equivalent resistance of inverter output filter, $\dot{U}_{\text {refers to positive }}$ sequence electromotive force. The calculation for threephase voltages $\dot{\mathrm{E}}_{\mathrm{a}}, \dot{\mathrm{E}}_{\mathrm{b}}, \dot{\mathrm{E}}_{\mathrm{c}}$ in the exit of inverter is as follows:

$$
\begin{gathered}
\dot{\mathrm{E}}_{\mathrm{a}} \dot{\mathrm{E}} \\
\dot{\mathrm{E}} \mathrm{e}^{\mathrm{j} 240^{\circ}} \dot{\mathrm{E}} \\
\dot{\mathrm{E}} \mathrm{e}^{\mathrm{j} 120^{\circ}} \dot{\mathrm{E}}
\end{gathered}
$$

The calculation formula of three-phase current value of inverter output is as follows:

$$
\begin{aligned}
& \dot{\mathrm{I}} \frac{\mathrm{E} a-U a}{\mathrm{a}+\mathrm{jX}} \\
& \dot{\mathrm{I}}_{\mathrm{b}} \frac{\mathrm{Eb}-\mathrm{Ub}}{\mathrm{R}+\mathrm{jX}}
\end{aligned}
$$

$$
\dot{\mathrm{I}} \frac{\mathrm{Ec}_{\mathrm{c}}-\mathrm{U}_{\mathrm{c}}}{\mathrm{R}+\mathrm{jX}}
$$

Through the above analysis, the output power of the inverter can be set unchangeably when the system malfunctions to ensure the unification of the front and back. In addition, there are low voltage protection devices in the inverter control device to protect the security of the system operation. When the voltage value of the inverter exit position is less than the set value, low voltage protection devices will make corresponding security protection action that is to shut off the power. This paper doesn't consider the effect of protection device in order to obtain the short circuit current value of power distribution system in the most serious situation[4].

According to the previous calculation law, if the power capacity exceeds the short-circuit capacity value of more than 3 times, it can be positioned as an infinite capacity of the power supply, in this way, the transient effects of the short circuit process will not be considered. However, the power supply capacity value is lower in the course of actual use, and at the same time, in order to increase the accuracy of short circuit calculation, the transient process of short circuit must be analyzed carefully. For the synchronous generator model, we assume that the numerical value of subtransient reactance of $\mathrm{d}$ axle and $\mathrm{q}$ axle is same. The damping winding calculation is as follows:

$$
\dot{\mathrm{E}}=\dot{\mathrm{U}}_{1}+\mathrm{j}_{1}+\mathrm{X}_{\mathrm{d}}{ }^{+}{ }_{1} \mathrm{R}_{\mathrm{a}}
$$

In the whole formula, $\dot{\mathrm{E}}_{\text {" refers to subtransient }}$ electromotive force, $\mathrm{R}_{\mathrm{a}}$ refers to synchronous generator stator equivalent resistance, $\dot{U}$ refers to terminal voltage of the synchronous generator, $\dot{U}$ refers to the output current of the synchronous generator. According to this situation, subtransient equivalent circuit diagram is drawn as follows [5].

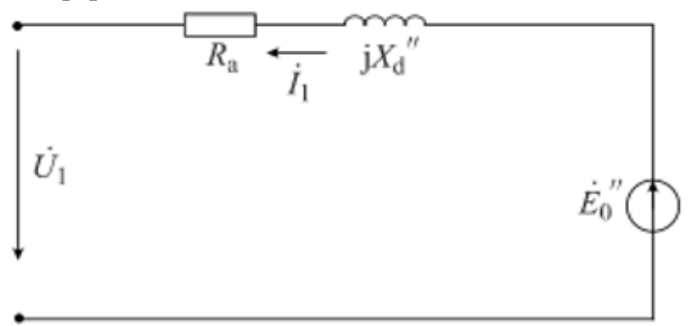

Figure 3. A subtransient equivalent circuit diagram

In this process, the subtransient electromotive force $\dot{E}_{" \prime}$ is in a steady state before and after the short circuit, so we can obtain it through the quantity of state calculated by steady state load flow calculation before fault see fig 3 .

$$
\dot{\mathrm{E}} \underset{0^{\prime \prime}=\mathrm{U}_{10}+\mathrm{j}_{10} \mathrm{X}_{\mathrm{d}}{ }^{\prime}+\mathrm{R}_{\mathrm{a}} 10}{\dot{\mathrm{I}}}
$$

Uं 10 refers to the terminal voltage of synchronous generator before short circuit, $\dot{I}_{10}$ is the corresponding output current. As for the value of three-phase current, the calculation is as follows: 


$$
\begin{aligned}
& \dot{I} \frac{E a-U a}{R a+j X d "} \\
& \dot{I} \underset{b=}{\frac{E b-U b}{R a+j X d "}} \\
& \dot{I} \underset{c=}{\dot{E}=} \frac{E c-U c}{R a+j X d "}
\end{aligned}
$$

In this process, firstly, we calculate the synchronous generator subtransient elecromotive force and then obtain the output current [6].

Asynchronous generator is a special case of a synchronous generator. The exciting voltage is constant and the numerical value is 0 . The parameter of $\mathrm{d}$ axle and $\mathrm{q}$ axle is unified and the revolving speed of them is not synchronous. The expression of subtransient reactance is as follows:

$$
\mathrm{X}^{\prime \prime}=\mathrm{X}_{\mathrm{a}^{\circ}}+\frac{\mathrm{Xr} \sigma \mathrm{Xad}}{\mathrm{Xr} \sigma+\mathrm{Xad}}
$$

In the formula, $X_{\mathrm{a \sigma}}$ refers to stator leakage reactance,

$\mathrm{Xr} \sigma$

refers to rotor leakage reactance value, Xad refers to direct axis armature reaction reactance. From the angle

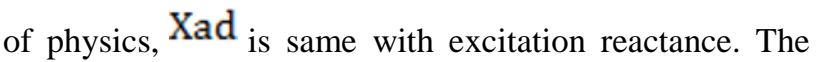
calculation of subtransient elecromotive force $\dot{E}_{0}$ " is as follows:

$$
\dot{\mathrm{E}}_{0^{\prime \prime}=\dot{\mathrm{U}}_{20}+\mathrm{j}_{20} \mathrm{X}^{\prime \prime}+\mathrm{R}_{\mathrm{a} 20}}^{\dot{\mathrm{I}}}
$$

$\dot{U}_{20}$ refers to the terminal voltage of asynchronous generator before short circuit, $\dot{\mathrm{I}}_{20}$ is the corresponding current.

\section{Conclusion}

The application of distributed power supply in the power system is very widespread. Depending on the different types of access systems, the corresponding calculation method should be adopted to obtain the short circuit current value, so as to provide protection for circuit security settings. In this paper, we summarize and analyze the above calculation. The optimization proposal is to adopt the combination of power flow calculation and short circuit calculation [7]. Firstly, we use the power flow calculation to obtain some state quantities, and then calculate the current. This method is practical, accurate and effective, which provides a guarantee for safe operation of the circuit.

\section{References}

1. Cheng-shan Wang, Xiao-qian Sun . The improving method of the distributed power distribution network short-circuit calculation [J]. Automation of electric power systems, 2012, 36 (23) : 54-58.

2. Shan Yang, Xiang-qian Tong, Jian Liu, etc. Including short circuit current calculation methods of distributed power distribution network study [J]. Power grid technology, 2015, 39 (7) : 1977-1982.

3. Manli Li, Hai-long Wang, Yang-Jiao Zhang. Containing distributed power distribution network adaptive protection method [J]. Electric power science and engineering, 2014 (5) : 62-66.

4. Xiang-gen Yin, Zhe Zhang, Fan Xiao, etc. Shortcircuit calculation model for distributed power supply and power grid fault calculation method research $[\mathrm{J}]$. Power system protection and control, 2015, 43 (22) : 1-9.

5. Ci Song, Li Hongjia, Chen Xin, et al. The cornerstone of energy internet : research and practice of distributed energy storage technology [J]. Science China: Information Sciences, 2014, 44(6): 762-773

6. Huang Ru, Ye Le, Liao Huailin. Microelectronics technologies in renewable energy internet[J]. Science China Information Sciences, 2014, 44(6): 728-742

7. Dong Chaoyang, Zhao Junhua, Wen Fushuan, et al. From Smart grid to energy internet:basic concept and research Framework[J]. Automation of Electric Power Systems, 2014, 38(15): 1 